\title{
ACTIVITY AS THE RESULT OF MERGING
}

\author{
V. M. KONTOROVICH, A. V. KATS and D. S. KRIVITSKY \\ Institute of Radio Astronomy of the Ukrainian Academy of Sciences \\ 4 Chervonopraporna str., 310002 Kharkiv, Ukraine
}

The observed correlation between activity and mergers of galaxies may be explained by the compensation of the angular momentum by merging. This leads to accretion on the galaxy centre [2].

The statistical description of the merger process is based on the generalized Smoluchowsky kinetic equation for the galaxy mass and angular momentum distribution $f(M, \mathbf{S}, t)[1]$. The model allows to find the luminosity function of active objects $\varphi(L, t)$ connected with $f(M, \mathbf{S}, t)$ by the quadratic in $f$ integral relation. It supposes the luminosity-mass excess $\Delta m$ relation: $L=B \cdot \Delta m$ (mass $\Delta m$ is able to fall to the centre). The simplest calculation scheme results in the function $\varphi \propto 1 / L$, close to the observed one, if the asymptotic expression of the mass function MF $f(M, t) \equiv \int f(M, \mathbf{S}, t) d \mathbf{S} \propto M^{\alpha}$ with $\alpha=-(u+2) / 2$, where $u$ is defined by the dependence of coalescence coefficient $U \propto M^{u}$ on mass. MF with $\alpha=-(u+2) / 2$ corresponds to the approximate conservation of the number of massive galaxies if their interaction with small ones (masses $\sim M_{*}$ ) prevails.

At $u>1$ (this value is typical for galaxies in the wide mass interval) the "explosive" evolution occurs - analog of the phase transition of gel formation [4], when the power-type tail of MF is formed during a finite time interval. Accordingly an "explosive" formation of active objects, i.e. the epoch of quasar formation, takes place [3]. On large enough masses $M_{f} \geq 10^{14} M_{\odot}$ the maximum value of the impact parameter is already limited by the mean free pass length or mean distance between the galaxies. So the mass dependence of the merger cross-section $\sigma$ and $U$ disappears $(u \rightarrow 0)$ and the "explosion" stops. This model (assuming an early emergence of small-mass galaxies $M_{*} \sim 10^{6} M_{\odot}$ ) enables to explain the abrupt disappearance of quasars at $z=z_{c r} \geq 3$.

The authors are grateful to ISF and the organizers of the IAU Symposium 159 for being given the opportunity to take part in the conference thanks to the Soros Travel Grant for one of the authors (V.M.K.).

\section{References}

1. Kats, A. V., and Kontorovich, V.M., 1990, JETP, 70,1; Pis'ma v. Astron. Zh., 17, 229; 1992, Astron. Astrophys. Trans., 2, 183.

2. Kontorovich, V. M., Kats, A. V. and Krivitsky, D. S., 1992, JETP Lett., 55, 1.

3. Stockmayer, W. H., 1943, J. Chem. Phys., 11, 45.

4. Toomre, A., and Toomre, J., 1972, Astrophys. J., 178, 623. 